\title{
马甲子叶中两个新化合物
}

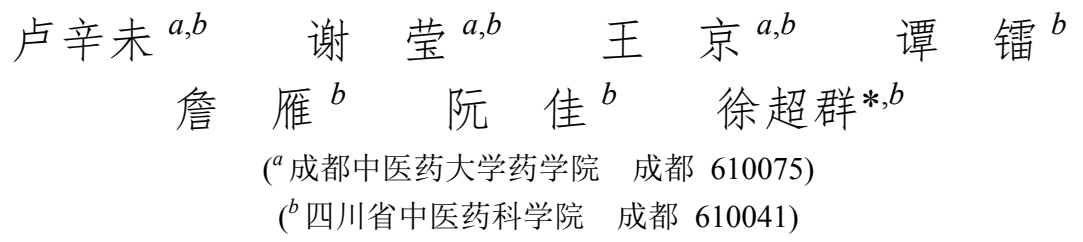

\begin{abstract}
摘要 研究马甲子 Paliurus ramosissimus (Lour.) Poir 叶子乙醇提取物的化学成分. 采用硅胶、高效液相制备色谱进行分 离纯化得到 6 个化合物，根据理化性质及 NMR、HR-ESIMS 等波谱技术鉴定上述化合物的结构分别为 $2 \alpha-O$-顺式对羟

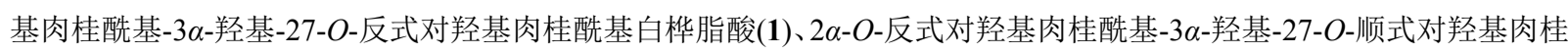
酰基白桦脂酸(2)、messagenic acid A (3)、messagenic acid B (4)、白桦脂醛(5)、 $\beta$-谷甾醇(6). 其中化合物 1 和 2 为新化 合物，命名为马甲子素 $\mathrm{A}$ 和马甲子素 $\mathrm{B}$, 属于白桦脂酸型五环三萜类化合物; 化合物 $\mathbf{3} \sim \mathbf{6}$ 首次从该植物中发现. 采用 四甲基偶氮唑蓝(MTT)法对化合物 1 3 进行体外抗肿瘤活性测试, 实验结果表明化合物 $\mathbf{1} \sim \mathbf{3}$ 具有显著的体外肿瘤细 胞生长抑制活性.
\end{abstract}

关键词 马甲子; 五环三萜；马甲子素 A；马甲子素 B；抗肿瘤活性

\section{Two New Compounds from the Leaves of Paliurus ramosissimus}

\author{
Lu, Xinwei ${ }^{a, b} \quad$ Xie, Ying ${ }^{a, b} \quad$ Wang, Jing ${ }^{a, b} \quad$ Tan, Lei $^{b} \quad$ Zhan, Yan $^{b}$ \\ Ruan, $\mathrm{Jia}^{b} \quad \mathrm{Xu}$, Chaoqun $*, b$ \\ ( ${ }^{a}$ School of Pharmacy, Chengdu University of Traditional Chinese Medicine, Chengdu 610075) \\ ( ${ }^{b}$ Institute of Pharmacy, Sichuan Academy of Chinese Medicine Sciences, Chengdu 610041)
}

\begin{abstract}
To study chemical constituents in the ethanol extract of Paliurus ramosissimus (Lour.) Poir, two new compounds along with four known compounds were isolated and purified by repeated gel column chromatographies and high performance preparative liquid chromatography. On the basis of spectroscopic methods (HR-ESIMS, 1D-and 2D-NMR), their structures were identified as $2 \alpha$-(cis-p-hydroxy-cinnamyloxy)-3 $\alpha$-hydroxy-27-(trans- $p$-hydroxy-cinnamyloxy)betulinic acid (1), $2 \alpha$ (trans- $p$-hydroxy-cinnamyloxy)-3 $\alpha$-hydroxy-27-(cis- $p$-hydroxy-cinnamyloxy)betulinic acid (2), messagenic acid A (3), messagenic acid B (4), betulinaldehyde (5) and $\beta$-sitosterol (6). Among them, compounds 1 and 2 were new compounds named as paliurusene $A$ and paliurusene $B$, which belong to triterpenoids, compounds $\mathbf{3} \sim \mathbf{6}$ were isolated from this plant for the first time. The anti-tumor activity of compounds $\mathbf{1} \sim \mathbf{3}$ was evaluated by methyl thiazolyl tetrazolium (MTT) assays. The result showed that compounds $\mathbf{1} \sim \mathbf{3}$ presented obvious cytotoxicity in vitro against the cancer cell lines.

Keywords Paliurus ramosissimus (Lour.) Poir.; pentacyclic triterpenoids; paliurusene A; paliurusene B; anti-tumor
\end{abstract}

马甲子 Paliurus ramosissimus (Lour.) Poir. 为鼠李科 马甲子属植物, 又名铁篱笆、一条龙、铜钱树, 系民间 常用草药, 在我国多省均有分布. 《中华本草》记载马 甲子叶具有清热解毒功效 ${ }^{[1]}$, 目前国内外对马甲子的化 学成分研究相对较少, 马甲子的根、茎的化学成分主要 有三萜类、环肽生物碱等 ${ }^{[2 \sim 6]}$, 马甲子果化学成分有三
萜类、香豆素类和二氢黄酮类等 ${ }^{[7,8]}$. 陈晨等从马甲子叶 乙醇提取物分离鉴定了 8 个化合物, 包括分别为 $3 \beta$-差弪 基-27-(3,4-二羟基)羽扇豆-20(29)-烯-28-酸、美洲茶酸、 羽扇豆醇等 ${ }^{[9]}$. 涉及生物活性的研究较少。韦国锋 等 ${ }^{[10,11]}$ 分别于 1998 年和 1999 年研究了马甲子水和 $95 \%$ 乙醇提取物的镇咳祛痰作用. 本课题组 ${ }^{[12]}$ 前期研究首

* Corresponding author. E-mail: chaoqun_xu@sina.com

Received July 29, 2016; revised September 22, 2016; published online October 11, 2016.

Project supported by the National Key Technology Research and Development Program of the Science and Technology Department of Sichuan Province (No. 15ZC1978), and the Sichuan Provincial Research Institute of Science and Technology Achievements Transformation Fund (No. 14010139).

四川省科技支撑计划(No. 15ZC1978)、四川省科研院所科技成果转化(No. 14010139)资助项目. 
次发现马甲子叶醇提物具有一定的体外抗肿瘤活性, 并 从马甲子叶醇提物中篮选分离得到了白桦脂酸和马甲 子素, 其中马甲子素为新的化合物, 属于白桦脂酸型五 环三萜类化合物, 具有一定的体外抗肿瘤活性 ${ }^{[13]}$. 为进 一步探寻马甲子叶中抗肿瘤活性成分, 本实验从马甲子 叶乙醇提取物中分离得到 6 个化合物, 分别鉴定为 $2 \alpha-O$-顺式对着基肉桂酰基- $3 \alpha$-羟基-27- $O$-反式对着基 肉桂酰基白桦脂酸(1)、2 $\alpha-O$-反式对羟基肉桂酰基- $3 \alpha-$ 羟基 -27- $O$ - 顺式对羟基肉桂酰基白桦脂酸 (2)、 messagenic acid A (3)、messagenic acid B (4)、白桦脂醛 (5)和 $\beta$-谷甾醇 (6)(图 1). 化合物 1,2 为新化合物, 命名 为马甲子素 $\mathrm{A}$ 和马甲子素 $\mathrm{B}$, 均属于白桦脂酸型五环三 萜类化合物. 化合物 $3 \sim 6$ 首次从本植物中发现. 化合物 1 3 表现出显著的体外抗肿瘤活性.

\section{1 结果与讨论}

化合物 1 为白色粉末, m.p. $201 \sim 203{ }^{\circ} \mathrm{C},[\alpha]_{\mathrm{D}}^{20}-$ 181.6 ( $c$ 0.33, MeOH). IR 光谱显示分子含有羟基(3389 $\left.\mathrm{cm}^{-1}\right)$ 和羰基 $\left(1702 \mathrm{~cm}^{-1}\right)$ 等官能团. ESI-MS m/z: 779.48 $[\mathrm{M}-\mathrm{H}]^{-}, 781.49[\mathrm{M}+\mathrm{H}]^{+}, 803.44[\mathrm{M}+\mathrm{Na}]^{+}, 819.50$ $[\mathrm{M}+\mathrm{K}]^{+}$. 显示该化合物的分子量为 780; HRESI-MS $m / z: 803.4126[\mathrm{M}+\mathrm{Na}]^{+}$(计算值 803.4135), 779.4164 $[\mathrm{M}-\mathrm{H}]^{-}$(计算值 779.4159), 确定其分子式为 $\mathrm{C}_{48} \mathrm{H}_{60} \mathrm{O}_{9}$. ${ }^{1} \mathrm{H}$ NMR (600 MHz, DMSO- $d_{6}$ ) 显示该化合物有 5 个甲基 信号 $\delta_{\mathrm{H}} 0.74,0.91,0.92,0.93,1.67$ (each s, 3H), 其中 $\delta_{\mathrm{H}}$ 1.67 为连接在双键上面的甲基氢信号. 在低场部分 $\delta_{\mathrm{H}}$ 7.72 (d, $J=8.4 \mathrm{~Hz}, 2 \mathrm{H}), 7.55$ (d, $J=8.4 \mathrm{~Hz}, 2 \mathrm{H}), 6.79$ (d, $J=8.4 \mathrm{~Hz}, 2 \mathrm{H}), 6.76(\mathrm{~d}, J=8.4 \mathrm{~Hz}, 2 \mathrm{H})$, 说明分子中有 两个苯环结构, 且苯环为对位取代, 同时在 $\delta_{\mathrm{H}} 7.53(\mathrm{~d}$, $J=15.6 \mathrm{~Hz}, 1 \mathrm{H}), 6.82$ (d, $J=12.6 \mathrm{~Hz}, 1 \mathrm{H}), 6.39$ (d, $J=$
$15.6 \mathrm{~Hz}, 1 \mathrm{H}), 5.74(\mathrm{~d}, J=12.6 \mathrm{~Hz}, 1 \mathrm{H})$ 为两个顺反结构双 键上面的氢信号. $\delta_{\mathrm{H}} 12.19,10.02,9.87(1 \mathrm{H}, \mathrm{s})$ 为分子中 的活泼氢信号. 结合 ${ }^{13} \mathrm{C} \mathrm{NMR}\left(150 \mathrm{MHz}, \mathrm{DMSO}-d_{6}\right)$ 和 DEPT(无畸变极化转移增强) $135^{\circ}$, 该化合物共有 48 个 碳原子信号, 在低场部分 $\delta_{\mathrm{C}} 177.7,167.1,166.1$ 为酯羰 基碳信号, $\delta_{\mathrm{C}} 160.3,159.2,150.5,126.0,125.4$ 为季碳信 号, $\delta_{\mathrm{C}} 145.0,143.3,133.2,130.8,116.6,116.2,115.3$, 114.9 为叔碳信号峰, $\delta_{\mathrm{C}} 110.2$ 为仲碳信号. 结合氢谱数 据，说明该化合物有两个对羟基肉桂酰基 ${ }^{[14]}$ ，根据它们 之间的偶合常数, 可以判断其中一个是反式结构，另一 个是顺式结构 ${ }^{[15]}$. 除此之外, 分子中还有一个双键. 在 DEPT $135^{\circ}$ 中, $\delta_{\mathrm{C}} 78.5,72.5$ 是连氧基团叔碳信号, $\delta_{\mathrm{C}} 62.5$ 是连氧基团仲碳信号. 除了两个对羟基肉桂酰基，该化 合物还剩 30 个碳信号, 再结合 ${ }^{1} \mathrm{H} \mathrm{NMR},{ }^{13} \mathrm{C} \mathrm{NMR}$ 和 DEPT $135^{\circ}$ 综合特征, 推断该化合物为 $\mathrm{C}_{30}$ 型三萜结构. 由 $\mathrm{HSQC}($ 异核单量子关系)对化合物的碳氢信号进行归 属, 两个对羟基肉桂酰基通过 $\operatorname{HMBC}\left({ }^{1} \mathrm{H}\right.$ 的异核多碳相 关谱)相关来确定. $\delta_{\mathrm{H}} 4.88\left(\delta_{\mathrm{C}} 72.5, \mathrm{C}-2\right)$ 与 $\delta_{\mathrm{C}} 166.1(\mathrm{C}-\gamma)$ 具有相关, $\delta_{\mathrm{H}} 4.51\left(\delta_{\mathrm{C}} 62.5, \mathrm{C}-27\right)$ 与 $\delta_{\mathrm{C}} 167.1\left(\mathrm{C}-\gamma^{\prime}\right)$ 具有相 关; 同时 $\delta_{\mathrm{H}} 5.74,6.82$ 与 $\delta_{\mathrm{C}} 166.1(\mathrm{C}-\gamma)$ 具有相关, $\delta_{\mathrm{H}} 7.53$, 6.39 与 $\delta_{\mathrm{C}} 167.1\left(\mathrm{C}-\gamma^{\prime}\right)$ 具有相关, 说明顺式对羟基肉桂酰 基是与母核 2 位碳相连，反式对着基肉桂酰基是与母核 27 位碳相连. $\delta_{\mathrm{H}} 1.67(\mathrm{H}-18), 1.82,1.38(\mathrm{H}-22)$ 与 $\delta_{\mathrm{C}} 177.7$ (C-28), 55.5 (C-17) HMBC 相关; $\delta_{\mathrm{H}} 4.71,4.58$ (H-29), $1.68(\mathrm{H}-30)$ 与 $\delta_{\mathrm{C}} 150.5(\mathrm{C}-20), 46.9(\mathrm{C}-19)$ 相关; $\delta_{\mathrm{H}} 0.91$ $(\mathrm{H}-23), 0.74(\mathrm{H}-24)$ 与 $\delta_{\mathrm{C}} 78.5(\mathrm{C}-3), 39.5(\mathrm{C}-4)$ 相关. 以上 进一步说明该化合物为 2- $O$-顺式对着基肉桂酰基-27- $O$ 反式对差基肉桂酰基白桦脂酸. 2,3 位取代基的相对构 型是由 NOESY(二维 NOE 谱)确定, 在 NOESY 谱图中 $\delta_{\mathrm{H}} 4.88(\mathrm{H}-2)$ 与 $\delta_{\mathrm{H}} 3.05(\mathrm{H}-3)$ 相关，同时 $\delta_{\mathrm{H}} 4.88(\mathrm{H}-2)$ 与
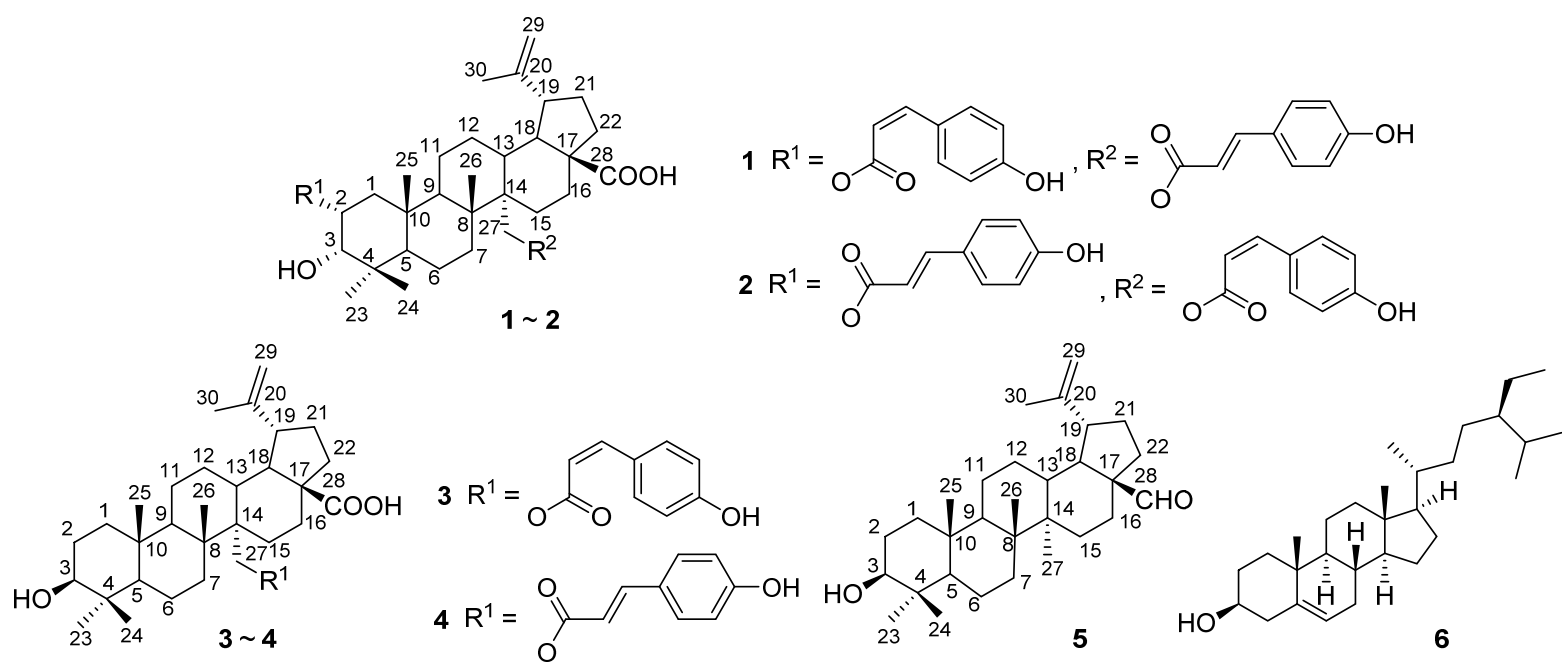

图 1 化合物 $1 \sim 6$ 的结构

Figure 1 Structures of compounds $1 \sim 6$ 
$\delta_{\mathrm{H}} 0.93(\mathrm{H}-25)$ 相关, $\delta_{\mathrm{H}} 3.05(\mathrm{H}-3)$ 与 $\delta_{\mathrm{H}} 0.74(\mathrm{H}-24)$ 相关, $\delta_{\mathrm{H}} 0.74(\mathrm{H}-24)$ 与 $\delta_{\mathrm{H}} 0.93(\mathrm{H}-25)$ 相关. 说明 2, 3 位取代基 均为 $\alpha$ 构型, 同样从 NOESY 图谱中可以看出 19 位取代 基为 $\alpha$ 构型.

综合以上结构分析，该化合物鉴定为 $2 \alpha-O$-顺式对 羟基肉桂酰基- $3 \alpha$-羟基-27- $O$-反式对羟基肉桂酰基白桦 脂酸. 经 Scifinder 检索, 未见有该化合物的相关报道, 确定化合物 1 为新三萜类结构, 命名为马甲子素 $\mathrm{A}$.

化合物 2 为白色无定性粉末, m.p. 202 203 ${ }^{\circ} \mathrm{C}$, $[\alpha]_{\mathrm{D}}^{20}-150.5(c 0.02, \mathrm{MeOH})$, ESI-MS 负离子 $m / z: 779.02$ $[\mathrm{M}-\mathrm{H}]^{-}$, 正离子 $m / z: 803.43[\mathrm{M}+\mathrm{Na}]^{+}$, 提示该化合物 的分子量为 780; HRESI-MS 给出 $m / z: 803.4139$ [M+ $\mathrm{Na}]^{+}$(计算值 803.4135), $779.4150[\mathrm{M}-\mathrm{H}]^{-}$(计算值 779.4159), 确定该化合物的分子式为 $\mathrm{C}_{48} \mathrm{H}_{60} \mathrm{O}_{9}$. 将化合 物 $\mathbf{2}$ 的 ${ }^{1} \mathrm{H}$ NMR, ${ }^{13} \mathrm{C}$ NMR (DMSO- $d_{6}$ ) 与化合物 $\mathbf{1}$ 进行比 较, 发现两者化学位移无明显区别, 说明化合物 2 同样 是二对羟基肉桂酰基取代白桦脂酸三萜类化合物. 结合 DEPT $135^{\circ}$, 通过 HSQC 及 HMBC 对该化合物进行碳氢 全归属; 利用 $\mathrm{HMBC}$ 对两个对羟基肉桂酰基的取代位 置进行确证: $\delta_{\mathrm{H}} 4.90(\mathrm{H}-3)$ 与 $\delta_{\mathrm{C}} 166.8(\mathrm{C}-\gamma)$ 具有远程相 关, $\delta_{\mathrm{H}} 7.54(\mathrm{H}-\alpha)$ 与 $\delta_{\mathrm{C}} 166.8(\mathrm{C}-\gamma), 115.5(\mathrm{C}-\beta), 125.6$ $\left(\mathrm{C}-1^{\prime}\right), 130.5\left(\mathrm{C}-2^{\prime}, 6^{\prime}\right)$ 相关, $\delta_{\mathrm{H}} 6.32(\mathrm{H}-\beta)$ 与 $\delta_{\mathrm{C}} 166.8(\mathrm{C}-\gamma)$, $144.5(\mathrm{C}-\alpha), 125.6\left(\mathrm{C}-1^{\prime}\right)$ 相关, 根据氢谱的偶合常数, 说 明反式对羟基肉桂酰基与母核 C-3 位相连; $\delta_{\mathrm{H}} 4.51,4.41$ $(\mathrm{H}-27)$ 与 $\delta_{\mathrm{C}} 166.6\left(\mathrm{C}-\gamma^{\prime}\right), 45.4(\mathrm{C}-14)$ 相关; $\delta_{\mathrm{H}} 6.87\left(\mathrm{H}-\alpha^{\prime}\right)$
与 $\delta_{\mathrm{C}} 166.6\left(\mathrm{C}-\gamma^{\prime}\right), 116.1\left(\mathrm{C}-\beta^{\prime}\right), 125.9\left(\mathrm{C}-1^{\prime \prime}\right), 132.8$ $\left(\mathrm{C}-2^{\prime \prime}, 6^{\prime \prime}\right)$ 具有远程相关, $\delta_{\mathrm{H}} 5.78\left(\mathrm{H}-\beta^{\prime}\right)$ 与 $\delta_{\mathrm{C}} 143.8\left(\mathrm{C}-\alpha^{\prime}\right)$, $166.6\left(\mathrm{C}-\gamma^{\prime}\right), 125.9\left(\mathrm{C}-1^{\prime \prime}\right)$ 具有相关, 根据氢谱化学位移 及偶合常数, 提示顺式对羟基肉桂酰基是连接在母核的 C-27 位上.

综合以上分析, 核磁数据与化合物 $\mathbf{1}$ 比较, 可以推 测化合物 $\mathbf{2}$ 与化合物 $\mathbf{1}$ 不同之处在于 2,27 位连接的对 着基肉桂酰基不同. 故化合物 2 鉴定为 $2 \alpha-O$-反式对羟 基肉桂酰基-3 $\alpha$-羟基-27-O-顺式对羟基肉桂酰基白桦脂 酸. 经 Scifinder 检索, 未见有该化合物的相关报道, 确 定化合物 2 为新三萜类结构, 命名为马甲子素 B.

化合物 3 6 为已知化合物, 经波谱分析及文献数 据对照, 将它们分别鉴定为 messagenic acid A (3) ${ }^{[16,17]}$ 、 messagenic acid B (4) ${ }^{[16,17]}$ 、白桦脂醛 $(5)^{[18]}$ 和 $\beta$-谷甾醇 $(6)^{[19]}$.

采用四甲基偶氮唑蓝(MTT)法检测化合物 $\mathbf{1} \sim \mathbf{3}$ 对 两株人肝脏肿瘤细胞(BEL7404 和 QGY7703)增值抑制 作用, 化合物 $\mathbf{1} \sim \mathbf{3}$ 与阳性对照药物氟腿嘧定(5-Fluorouracil)相比, 均有显著体外肿瘤细胞生长抑制活性, 其对不同细胞的 $\mathrm{IC}_{50}$ 值见表 2 .

\section{2 实验部分}

\section{1 仪器与试剂}

柱色谱硅胶(100 200, 300 400 目)和薄层色谱硅
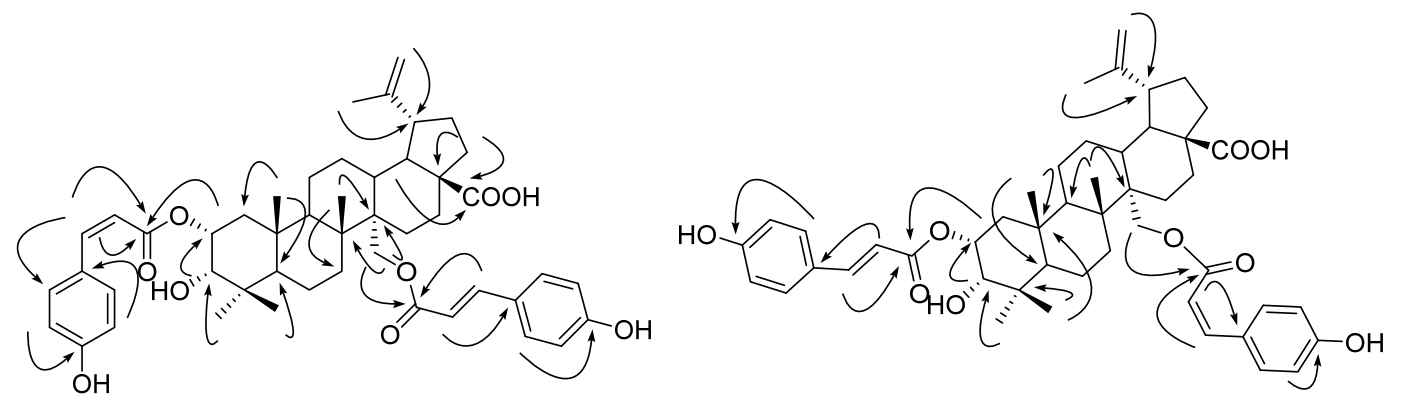

图 2 化合物 $\mathbf{1}$ 和 $\mathbf{2}$ 的主要 $\mathrm{HMBC}$ 相关

Figure 2 Key HMBC correlations for compounds $\mathbf{1}$ and $\mathbf{2}$
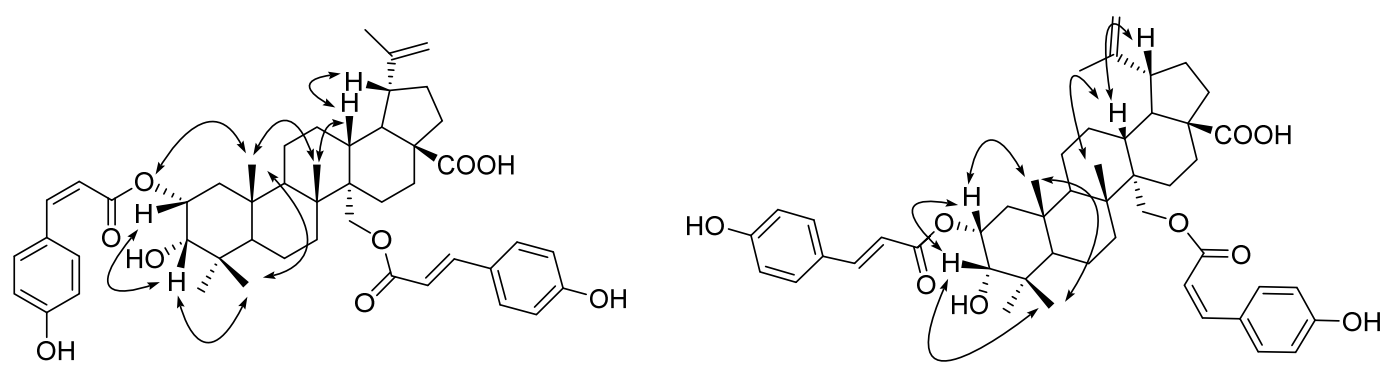

图 3 化合物 $\mathbf{1}$ 和 $\mathbf{2}$ 的主要 NOSEY 相关

Figure 3 Key NOSEY correlations for compounds $\mathbf{1}$ and $\mathbf{2}$ 
表 1 化合物 1 和 $\mathbf{2}$ 的碳氢归属(DMSO- $\left.d_{6}\right)$

Table $1{ }^{1} \mathrm{H}$ NMR $(600 \mathrm{MHz})$ and ${ }^{13} \mathrm{C}$ NMR $(150 \mathrm{MHz})$ data of compounds $\mathbf{1}$ and 2 (DMSO- $d_{6}$ )

\begin{tabular}{|c|c|c|c|c|}
\hline \multirow{2}{*}{ No. } & \multicolumn{2}{|c|}{ Compound 1} & \multicolumn{2}{|c|}{ Compound 2} \\
\hline & $\delta_{\mathrm{C}}(150 \mathrm{MHz})$ & $\delta_{\mathrm{H}}(600 \mathrm{MHz})$ & $\delta_{\mathrm{C}}(150 \mathrm{MHz})$ & $\delta_{\mathrm{H}}(600 \mathrm{MHz})$ \\
\hline 1 & $44.0 \mathrm{t}$ & $1.94 \mathrm{~m}, 0.94 \mathrm{~m}$ & $44.2 \mathrm{t}$ & $1.93 \mathrm{~m}, 0.96 \mathrm{~m}$ \\
\hline 2 & $72.5 \mathrm{~d}$ & $4.88 \mathrm{~m}$ & $72.6 \mathrm{~d}$ & $4.90 \mathrm{~m}$ \\
\hline 3 & $78.5 \mathrm{~d}$ & $3.05 \mathrm{dd}, J=9.6,6.0 \mathrm{~Hz}$ & $78.6 \mathrm{~d}$ & $3.11 \mathrm{~d}, J=9.6 \mathrm{~Hz}$ \\
\hline 4 & $39.5 \mathrm{~s}$ & & $39.5 \mathrm{~s}$ & \\
\hline 5 & $54.8 \mathrm{~d}$ & $0.88 \mathrm{~m}$ & $54.9 \mathrm{~d}$ & $0.87 \mathrm{~m}$ \\
\hline 6 & $18.4 \mathrm{t}$ & $1.43 \mathrm{~m}, 1.35 \mathrm{~m}$ & $18.4 \mathrm{t}$ & $1.45 \mathrm{~m}, 1.35 \mathrm{~m}$ \\
\hline 7 & $35.0 \mathrm{t}$ & $1.49 \mathrm{~m}, 1.42 \mathrm{~m}$ & $35.0 \mathrm{t}$ & $1.40 \mathrm{~m}, 1.36 \mathrm{~m}$ \\
\hline 8 & $41.5 \mathrm{~s}$ & & $41.5 \mathrm{~s}$ & \\
\hline 9 & $51.2 \mathrm{~d}$ & $1.40 \mathrm{~m}$ & $51.2 \mathrm{~d}$ & $1.38 \mathrm{~m}$ \\
\hline 10 & $38.6 \mathrm{~s}$ & & $38.6 \mathrm{~s}$ & \\
\hline 11 & $21.2 \mathrm{t}$ & $1.38 \mathrm{~m}, 1.17 \mathrm{~m}$ & $21.1 \mathrm{t}$ & $1.36 \mathrm{~m}, 1.17 \mathrm{~m}$ \\
\hline 12 & $25.1 \mathrm{t}$ & $1.66 \mathrm{~m}, 0.87 \mathrm{~m}$ & $25.1 \mathrm{t}$ & $1.65 \mathrm{~m}, 0.86 \mathrm{~m}$ \\
\hline 13 & $38.8 \mathrm{~d}$ & $2.40 \mathrm{dd}, J=12.6,2.4 \mathrm{~Hz}$ & $38.7 \mathrm{~d}$ & $2.40 \mathrm{t}, J=11.4 \mathrm{~Hz}$ \\
\hline 14 & $45.6 \mathrm{~s}$ & & $45.4 \mathrm{~s}$ & \\
\hline 15 & $24.0 \mathrm{t}$ & $1.75 \mathrm{~m}, 1.28 \mathrm{~m}$ & $24.0 \mathrm{t}$ & $1.60 \mathrm{~m}, 1.25 \mathrm{~m}$ \\
\hline 16 & $32.5 \mathrm{t}$ & $2.14 \mathrm{~m}, 1.18 \mathrm{~m}$ & $32.5 \mathrm{t}$ & $2.06 \mathrm{~m}, 1.11 \mathrm{~m}$ \\
\hline 17 & $55.5 \mathrm{~s}$ & & $55.5 \mathrm{~s}$ & \\
\hline 18 & $49.2 \mathrm{~d}$ & $1.67 \mathrm{~m}$ & $49.1 \mathrm{~d}$ & $1.63 \mathrm{~m}$ \\
\hline 19 & $46.9 \mathrm{~d}$ & $2.97 \mathrm{~m}$ & $46.9 \mathrm{~d}$ & $2.96 \mathrm{~m}$ \\
\hline 20 & $150.5 \mathrm{~s}$ & & $150.5 \mathrm{~s}$ & \\
\hline 21 & $30.4 \mathrm{t}$ & $1.82 \mathrm{~m}, 1.32 \mathrm{~m}$ & $30.5 \mathrm{t}$ & $1.81 \mathrm{~m}, 1.31 \mathrm{~m}$ \\
\hline 22 & $36.6 \mathrm{t}$ & $1.81 \mathrm{~m}$ & $36.6 \mathrm{t}$ & $1.81 \mathrm{~m}$ \\
\hline 22 & $30.0 t$ & $1.38 \mathrm{~m}$ & 50.01 & $1.35 \mathrm{~m}$ \\
\hline 23 & $28.8 \mathrm{q}$ & $0.91 \mathrm{~s}$ & $28.8 \mathrm{q}$ & $0.92 \mathrm{~s}$ \\
\hline 24 & $17.3 \mathrm{q}$ & $0.74 \mathrm{~s}$ & $17.3 \mathrm{q}$ & $0.74 \mathrm{~s}$ \\
\hline 25 & $17.8 \mathrm{q}$ & $0.93 \mathrm{~s}$ & $17.8 \mathrm{q}$ & $0.93 \mathrm{~s}$ \\
\hline 26 & $16.5 \mathrm{q}$ & $0.92 \mathrm{~s}$ & $16.5 \mathrm{q}$ & $0.90 \mathrm{~s}$ \\
\hline 27 & $62.5 \mathrm{t}$ & $4.51 \mathrm{q}, J=12.6 \mathrm{~Hz}$ & $62.5 \mathrm{t}$ & $\begin{array}{l}4.54 \mathrm{~d}, J=12.6 \mathrm{~Hz} \\
4.41 \mathrm{~d}, J=12.6 \mathrm{~Hz}\end{array}$ \\
\hline 28 & $177.7 \mathrm{~s}$ & & $177.8 \mathrm{~s}$ & \\
\hline 29 & $110.2 \mathrm{t}$ & $4.71 \mathrm{~s}, 4.58 \mathrm{~s}$ & $110.1 \mathrm{t}$ & $4.69 \mathrm{~s}, 4.57 \mathrm{~s}$ \\
\hline 30 & $19.4 \mathrm{q}$ & $1.68 \mathrm{~s}$ & $19.5 \mathrm{q}$ & $1.68 \mathrm{~s}$ \\
\hline$\alpha$ & $143.3 \mathrm{~d}$ & $6.82 \mathrm{~d}, J=12.6 \mathrm{~Hz}$ & $144.5 \mathrm{~d}$ & $7.54, \mathrm{~d}, J=15.6 \mathrm{~Hz}$ \\
\hline$\beta$ & $116.6 \mathrm{~d}$ & $5.74 \mathrm{~d}, J=12.6 \mathrm{~Hz}$ & $115.5 \mathrm{~d}$ & $6.32, \mathrm{~d}, J=15.6 \mathrm{~Hz}$ \\
\hline$\gamma$ & $166.1 \mathrm{~s}$ & & $166.8 \mathrm{~s}$ & \\
\hline $1^{\prime}$ & $126.0 \mathrm{~s}$ & & $125.6 \mathrm{~s}$ & \\
\hline $2^{\prime}, 6^{\prime}$ & $133.2 \mathrm{~d}$ & $7.71 \mathrm{~d}, J=8.4 \mathrm{~Hz}$ & $130.5 \mathrm{~d}$ & $7.51 \mathrm{~d}, J=8.4 \mathrm{~Hz}$ \\
\hline $3^{\prime}, 5^{\prime}$ & $116.2 \mathrm{~d}$ & $6.79 \mathrm{~d}, J=8.4 \mathrm{~Hz}$ & $115.4 \mathrm{~d}$ & $6.76 \mathrm{~d}, J=8.4 \mathrm{~Hz}$ \\
\hline $4^{\prime}$ & $160.3 \mathrm{~s}$ & & $159.3 \mathrm{~s}$ & \\
\hline$\alpha^{\prime}$ & $145.0 \mathrm{~d}$ & $7.53, \mathrm{~d}, J=15.6 \mathrm{~Hz}$ & $143.8 \mathrm{~d}$ & $6.87 \mathrm{~d}, J=12.6 \mathrm{~Hz}$ \\
\hline$\beta^{\prime}$ & $114.9 \mathrm{~d}$ & $6.39 \mathrm{~d}, J=15.6 \mathrm{~Hz}$ & $116.1 \mathrm{~d}$ & $5.78 \mathrm{~d}, J=12.6 \mathrm{~Hz}$ \\
\hline$\gamma^{\prime}$ & $167.1 \mathrm{~s}$ & & $166.6 \mathrm{~s}$ & \\
\hline $1 "$ & $125.4 \mathrm{~s}$ & & $125.9 \mathrm{~s}$ & \\
\hline $2 ", 6 "$ & $130.8 \mathrm{~d}$ & $7.55 \mathrm{~d}, J=8.4 \mathrm{~Hz}$ & $132.8 \mathrm{~d}$ & $7.65 \mathrm{~d}, J=8.4 \mathrm{~Hz}$ \\
\hline 3",5" & $115.3 \mathrm{~d}$ & $6.76 \mathrm{~d}, J=8.4 \mathrm{~Hz}$ & $116.2 \mathrm{~d}$ & $6.80 \mathrm{~d}, J=8.4 \mathrm{~Hz}$ \\
\hline $4 "$ & $159.2 \mathrm{~s}$ & & $160.2 \mathrm{~s}$ & \\
\hline
\end{tabular}

胶(青岛海洋化工厂产品); Bruker AV II-400 (600) MHz 核磁共振仪(TMS 为内标, 瑞士 Bruker 公司); 美国赛默 飞尼高力红外光谱仪 Nicolet iS50; Quattro Premier XE 质谱仪(美国 Waters 公司); Q-TOF Premier 高分辨质谱仪
(美国 Waters 公司); 轴向压缩柱 100 系统 APS-2004(北 京创新通恒科技有限公司); ultimate xb-c18(美国 Welch 公司); Sartorius BS210S 电子天平(北京赛多利斯天平有 限公司); Mettler Toledo 超越系列专业型 XS 分析天平(梅 
表 2 化合物 $1 \sim 3$ 对不同细胞的 $\mathrm{IC}_{50}$ 值 $(\mu \mathrm{mol} / \mathrm{L})$

Table $2 \mathrm{IC}_{50}(\mu \mathrm{mol} / \mathrm{L})$ of compounds $\mathbf{1} \sim \mathbf{3}$ against different cell lines

\begin{tabular}{ccc}
\hline Compd. & BEL7404 & QGY7703 \\
\hline $\mathbf{1}$ & 3.87 & 5.44 \\
$\mathbf{2}$ & 2.50 & 4.23 \\
$\mathbf{3}$ & 5.49 & 13.88 \\
5-Fluorouracil & 32 & 35 \\
\hline
\end{tabular}

特勒-托利多有限公司); XMTD-8222 真空干燥箱(上海 精宏实验设备有限公司); FZ102 粉碎机(北京中兴伟业 仪器有限公司); 1200 高效液相色谱仪(美国安捷伦公 司). 试剂均为分析纯. DMEM 培养基, 胎牛血清 (Hyclone, 美国)，3-(4,5-二甲基噻唑-2)-2,5-二苯基四氮 唑溴盐(MTT), 青霉素、链霉素(Amresco, 美国), 胰蛋白 酶(Gibco, 美国), 人肝癌细胞 BEL7404, QGY7703(四川 大学华西药学院靶向药物及释药系统教育部重点实验 室提供). 5-FU 注射液 (天津金耀氨基酸有限公司). Thermo Forma 系列 $\mathrm{CO}_{2}$ 培养箱(Sanyo, 日本); Model-550 型酶联免疫检测仪(Bio-Rad, 美国); SW-CJ-2D 型 超净工作台(苏州市净化设备总厂); 倒置显微镜(型号 Fx-35DX, Nikon); 离心机(型号 TGL-16G, 上海医用分 析仪器厂).

\section{2 药材标本}

实验用马甲子(多年生)于 2013 年 7 月采自四川省双 流县牧马山, 经四川省中医药科学院舒光明研究员鉴定 为鼠李科马甲子属植物马甲子 Paliurus ramosissimus (Lour.) Poir..

\section{3 提取分离}

取马甲子 $20 \mathrm{~kg}$ 粉碎，用 $95 \%$ 乙醇 10 倍量浸泡 $3 \mathrm{~d}$, 收集浸提液, 减压浓缩得浸膏约 $1.5 \mathrm{~kg}$. 总浸膏用水分 散后依次用石油醚、乙酸乙酯和正丁醇萃取, 得到乙酸 乙酯萃取物 $450 \mathrm{~g}$. 再将其经硅胶柱色谱分离, 采用氯 仿一甲醇 $(V: V=100: 0 \rightarrow 0 ： 100)$ 梯度洗脱, 薄层色谱 检测得到 7 个流分(Fr.A Fr.G). Fr.F(约 38 g)经 MCI 柱 分离, 乙醇-水系统 $(50 \% \rightarrow 95 \%)$ 依次洗脱, 高效液相色

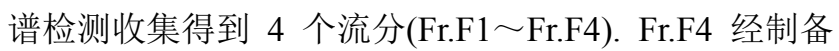
HPLC(流动相 $63 \%$ 乙腈水溶液)纯化得化合物 1 (25.1 $\mathrm{mg}, t_{\mathrm{R}}=22.5 \mathrm{~min}$ ). Fr.F4 制备流出液经制备 HPLC(流动 相 $62 \%$ 乙腈水溶液)得化合物 $4\left(15.5 \mathrm{mg}, t_{\mathrm{R}}=26.0 \mathrm{~min}\right)$. Fr.F4 制备流出液经制备 HPLC(流动相 $63 \%$ 乙腈水溶液) 得化合物 3 (22.2 mg, $\left.t_{\mathrm{R}}=25.5 \mathrm{~min}\right)$. Fr.F4 制备流出液经 制备 HPLC(流动相 $63 \%$ 乙腈水溶液)得化合物 2 (24.3 $\left.\mathrm{mg}, t_{\mathrm{R}}=25.5 \mathrm{~min}\right)$. Fr.B(约 $24 \mathrm{~g}$ ) 经硅胶柱层析, 采用氯 仿-甲醇 $(V: V=100: 1 \rightarrow 1: 1)$ 梯度洗脱, 得到 4 个部 分(Fr.B1 Fr.B4). Fr.B4 经反复硅胶柱层析, 得到化合物
$5(46.1 \mathrm{mg})$ 和 6 (35.0 mg).

\section{4 抗肿瘤活性测试}

按文献[20]方法复苏 BEL7404 细胞和 QGY7703 细 胞. 取对数生长期细胞, 用含 $10 \%$ 胎牛血清的改良杜氏 伊格尔培养基制成 $1 \times 105$ 个 $/ \mathrm{mL}$ 单细胞悬液，接种于 96 孔板中, 置 $37{ }^{\circ} \mathrm{C}, 5 \% \mathrm{CO}_{2}$ 培养箱培养至细胞贴壁后, 加入不同浓度的化合物 $1 \sim 3$ 和 5-Fu 注射液(阳性对照), 每组设复孔 4 个, 同时设立阴性对照、空白对照组, 继 续培养 $72 \mathrm{~h}$. 吸去上清液, 用含 $0.5 \mathrm{mg} / \mathrm{mL} \mathrm{MTT}$ 的无血 清 DMEM 孵育 $3 \mathrm{~h}$ 后, 弃上清液, 每孔加入 $150 \mu \mathrm{L}$ $\mathrm{DMSO}$, 在酶标仪上于 $490 \mathrm{~nm}$ 处检测各吸光度 $(A)$ 值. 实验重复 3 次. 按下列公式计算细胞生长抑制率，通过 spss 软件 ${ }^{[21]}$ 计算 $\mathrm{IC}_{50}$ 值.

$$
\begin{aligned}
& \text { 抑制率 }(\%)=1-(A \text { 实验组 } \\
& A \text { 空白对照组 }) /(A \text { 阴栍对照经 }) \times 100 \%
\end{aligned}
$$

辅助材料(Supporting Information) 新化合物 1 的红外 光谱，新化合物 1 和 2 的质谱、高分辨质谱、1D/2D NMR 谱图. 这些材料可以免费从本刊网站(http://sioc-journal. $\mathrm{cn} /)$ 上下载.

\section{References}

[1] Chinese Materia Medica, Shanghai Science and Technology Press, Shanghai, 1999, p. 242 (in Chinese). (中华本草，上海科学技术出版社，上海, 1999, p. 242.)

[2] Lee, S. S.; Su, W. C.; Liu, K. C. Nat. Prod. 1991, 54, 615.

[3] Lee, S. S.; Lin, C. J.; Liu, K. C. Nat. Prod. 1992, 55, 602.

[4] Lin, H. Y.; Chen, C. H.; Liu, K. C.; Lee, S. S. Helv. Chim. Acta 2003, 86, 127.

[5] Lin, H. Y.; Chen, C. H.; You, B. J.; Liu, K. C.; Lee, S. S. Nat. Prod. 2000, 63, 1338.

[6] Lee, S. S.; Su, W. C.; Liu, K. C. S. C. Phytochemistry 2001, 58, 1271.

[7] Yu, L.; Zhang, D. M. China J. Chin. Mater. Med. 2006, 31, 2049 (in Chinese).

(于否，张东明，中国中药杂志, 2006, 31, 2049.)

[8] Yu, L.; Yang, J. Z.; Zhang, D. M. Acta Pharm. Sin. 2009, 44, 625 (in Chinese). (于否, 杨敬芝, 张东明, 药学学报, 2009, 44, 625.)

[9] Chen, C.; Luo, G. D.; Hu, R.; Fu, H. Z. J. Chin. Pharm. Sci. 2016, 111.

[10] Wei, G. F.; Qin, D. G.; Huang, Z. W. J. Math. Med. 1999, 12, 165 (in Chinese). (韦国锋, 覃道光, 黄志文, 数理医药学杂志, 1999, 12, 165.)

[11] Wei, G. F.; Qin, D. G.; Huang, Z. W. J. Youjiang Med. Coll. Nationalities 1998, 20, 176 (in Chinese). (韦国锋, 覃道光, 黄志文, 右江民族医学院学报, 1998, 20, 176.)

[12] Gao, Y.; Song, L. Q.; Fan, M.; Yuan, Z. X.; Yu, Y.; Xu, C. Q. West China J. Pharm. Sci. 2015, 3, 303 (in Chinese). (高媛, 宋联强, 樊梅, 袁志翔, 余悦, 徐超群, 华西药学杂志, 2015, 3, 303.)

[13] Wu, R. J.; Song, L. Q.; Tan, L.; Zhan, Y.; Ruan, J.; Xu, C. C. Chin Tradit. Herb. Drugs 2015, 19, 2834 (in Chinese). 
(武芯娟, 宋联强, 谭镭, 詹雁, 阮佳, 中草药, 2015, 19, 2834.)

[14] Wu, L. J.; Xiang, T.; Liu, T. H.; Tian, Y.; Wang, Y. Q.; Gao, Z. Y.; Chen, L. Chin Tradit. Herb. Drugs 1998, 6, 367 (in Chinese). (吴立军, 相婷, 刘铁汉, 田燕, 王永奇, 高之杨, 陈莉, 中草药, 1998, 6, 367.)

[15] Wang, X. X.; Gao, H. Y.; Jiang, Y.; Zhao, M. B.; Wu, L. J.; Tu, P. F. J. Chin Tradit. Herb. Drugs 2013, 10, 1241 (in Chinese). (王晓霞, 高慧媛, 姜勇, 赵明波, 吴立军, 屠鹏飞, 中草药, 2013, 10, 1241.)

[16] Cortez, D. A. G.; Young, M. C. M.; Marston, A. Phytochemistry 1998, 47, 1367.

[17] Suksamrarn, A.; Tanachatchairatana, T.; Kanokmedhakul, S. J. Ethnopharmacol. 2003, 88, 275.
[18] Theerachayanan, T.; Sirithunyalug, B.; Piyamongkol, S. CMU J. Nat. Sci. 2007, 6, 253.

[19] Xu, R. S.; Yuan, K.; Yin, M. W.; Fang, L. J.; Mao, N. N. Chin Tradit. Herb. Drugs 2009, 7, 1015 (in Chinese).

(徐润生, 袁珂, 殷明文, 方否杰, 毛浓浓, 中草药, 2009, 7, 1015.)

[20] Situ, Z. Q.; Wu, J. Z. Cell Culture, World Book Inc, Xi'an, 2007, p. 79 (in Chinese).

(司徒镇强, 吴军正, 细胞培养, 世界图书出版公司, 西安, 2007, p. 79.)

[21] Zhou, Y. P. Prog. Pharm. Sci. 2003, (5), 314 (in Chinese). (周一平, 药学进展, 2003, (5), 314.) 